\title{
Los empresarios frente al narcotráfico en México. Tratamiento informativo de las reacciones empre- sariales ante situaciones de violencia e inseguridad
}

\author{
Carlos MuÑIZ \\ carlos.munizm@uanl.mx \\ Universidad Autónoma de Nuevo León (México) \\ Jacobo RAMÍREZ \\ jra.ikl@cbs.dk \\ Copenhagen Business School (Dinamarca)
}

Recibido: 7 de mayo de 2014

Aceptado: 2 de septiembre de 2014

\section{Resumen}

Los niveles de violencia e inseguridad en México han aumentado desde el inicio de la "guerra contra el narcotráfico" en 2006. En este artículo se busca conocer el tratamiento informativo que se ha prestado a este hecho en la prensa, en especial respecto de los actos delictivos cometidos sobre los pequeños y medianos empresarios del país y sus negocios o Pymes. Se realizó un análisis de contenido de noticias de prensa proveniente de Estados Unidos (EE.UU.), México y la Unión Europea (UE). Los resultados muestran que los periódicos de EE.UU y la UE presentan más noticias que los mexicanos. Además, domina la cobertura de las acciones violentas sobre los propios empresarios, principalmente desde un encuadre noticioso de conflicto en la UE, de consecuencias económicas en EE.UU. y moralidad en México.

Palabras clave: Tratamiento informativo, violencia, inseguridad, México, empresarios.

\section{Entrepreneurs against Drug Trafficking in Mexico. Framing of Entrepreneurs' Reactions to Violence and Insecurity Situations}

\begin{abstract}
The levels of violence and insecurity in Mexico have increased since the implementation of the "war against drug cartels" in 2006. This article seeks to identify the press framing of criminal acts against entrepreneurs and their small and medium-sized enterprises (SMEs) in Mexico. A content analysis was performed on press news from United States (U.S.), Mexico and the European Union (EU). The results show that the newspapers from the U.S. and the EU have more news than the Mexicans. The results also show that violent acts towards entrepreneurs are covered using the conflict frame in the EU, economic consequences frame in the U.S. and morality frame in Mexico.
\end{abstract}

Keywords: Framing, violence, insecurity, Mexico, entrepreneurs.

\section{Referencia normalizada}

MUÑIZ, Carlos y RAMÍREZ, Jacobo (2015): “Los empresarios frente al narcotráfico en México. Tratamiento informativo de las reacciones empresariales ante situaciones de violencia e inseguridad". Estudios sobre el Mensaje Periodístico. Vol. 21, Núm. 1 (enero-junio), págs.: 437-453. Madrid, Servicio de Publicaciones de la Universidad Complutense.

Sumario: 1. Introducción. 2. Marco teórico; 2.1. La violencia e inseguridad en México y la respuesta empresarial; 2.2. Framing de la violencia e inseguridad en México. 3. Método; 3.1. Corpus de noticias analizado; 3.2. Libro de códigos; 3.3. Procedimiento y fiabilidad. 4. Análisis de los resultados. 5. Discusión y conclusiones. 6 . Referencias bibliográficas.

\section{Introducción}

En diciembre de 2006 Felipe Calderón comenzó su administración como Presidente de México, la cual estuvo marcada desde el inicio por la que ha sido denominada 
como "guerra contra el narcotráfico", una estrategia iniciada por el mandatario pocos días después de asumir el cargo cuando presentó la "Operación Conjunta Michoacán". Ello se tradujo en el empleo de acciones de fuerza, incluso con el uso del ejército, contra los cárteles del narcotráfico que operaban en el país (Campbell y Hansen, 2014; Correa-Cabrera, 2012; Hernández y Rodelo, 2010). Desde el inicio de esta "guerra" los niveles de inseguridad aumentaron drásticamente en México, manifestándose en aumento de actos como secuestros, robos y asesinatos relacionados con las drogas (Carreón y de la Cruz, 2012; Rios, 2014). Hasta tal punto de que se calcula que durante el sexenio del ex-Presidente Felipe Calderón, que transcurrió entre 2006 y 2012, se produjeron entre 60,000 y 100,000 muertes relacionadas directamente con la citada guerra contra los cárteles de la droga y las actividades relacionadas con los mismos (Campbell y Hansen, 2014; Magaloni et al., 2014).

Dentro de las acciones violentas que la guerra contra el narco ha dejado en México se encuentran el asalto con arma, las violaciones, los secuestros y los homicidios, sufridos por la ciudadanía en general (Magaloni et al., 2014), así como la extorsión y el pago del denominado "derecho de piso", que afecta sobre todo a empresarios, en especial a aquellos que cuentan con mayor debilidad como son los pequeños y medianos empresarios y sus negocios o Pymes (Carreón y de la Cruz, 2012). Desde 2008 las extorsiones a comercios y empresarios aumentaron (Correa-Cabrera, 2012), constituyéndose de hecho en uno de los delitos más extendido (Rios, 2014). Ante este clima sufrido por la sociedad en general, y el empresariado en particular, no es de extrañar que hayan aumentado las tasas de emigración dentro de México y hacia los Estados Unidos (EE.UU.) (Durin, 2012). Se estima que son unos 115.000 los mexicanos que han llegado a ciudades fronterizas del sur de EE.UU. desde 2006, en muchos casos junto con sus negocios que son reabiertos en el nuevo destino (Rios, 2014).

Los medios de comunicación, tanto nacionales mexicanos como en el ámbito internacional, dieron sobrada cobertura de esta "guerra contra el narcotráfico". Se hicieron habituales en los medios informaciones sobre secuestros, asesinatos y demás acciones violentas sufridas por la ciudadanía mexicana y sus diferentes grupos sociales (Correa-Cabrera, 2012; Rodelo, 2014). A pesar de este especial papel jugado por los medios, aún son pocos los estudios sobre la cobertura informativa de tales eventos provenientes de México (Rodelo, 2014), y menos los que se hayan centrado en la realidad de un grupo social de especial importancia para el país como son los pequeños empresarios y sus empresas (Pymes). En este sentido, el presente trabajo busca explorar la cobertura realizada por la prensa internacional de la violencia relacionada con el narcotráfico en México, en especial aquella que afecta a los pequeños empresarios, así como sus respuestas o reacciones a estas presiones recibidas.

Para ello, se realizó un análisis de contenido partiendo de la Teoría del Framing como marco de referencia (de Vreese, 2003; Entman, 1993; Semetko y Valkenburg, 2000), pues permite estudiar la construcción de una narrativa de un conflicto internacional, la que puede determinar las percepciones públicas que se tienen sobre otros países e incluso las decisiones de inversión en países externos por parte del empresariado (Muñiz et al., 2009). No en vano, muchas empresas extranjeras han retrasado o cancelado planes de inversión directa en México debido a la violencia organizada en el 
país (American Chamber of Commerce of Mexico, 2010). Por ello, el estudio también se planteó como objetivo determinar las diferencias o similitudes en la cobertura del asunto realizada por tres países o regiones, como son EE.UU., México y la Unión Europea (UE), entre quienes existen lazos culturales y comerciales, como el Tratado de Libre Comercio de América del Norte (TLCAN) y la Asociación Económica, Concertación Política y Cooperación (Acuerdo Global) entre México y la UE.

\section{Marco teórico}

\subsection{La violencia e inseguridad en México y la respuesta empresarial}

Cuando el 11 de diciembre de 2006 Felipe Calderón, días después de dar inicio a su mandato, anunció el inicio de la "Operación Conjunta Michoacán", se abrió una etapa en el país marcada por el aumento considerable en los niveles de violencia e inseguridad (Correa-Cabrera, 2012; Magaloni et al., 2014) en lo que se ha denominado como "guerra contra el narcotráfico". Ello debido a las acciones contra los integrantes de las organizaciones de tráfico de drogas que operan en México y se encargan del tráfico de droga principalmente hacia los EE.UU. (Campbell y Hansen, 2014). Este anuncio del ex-Presidente se circunscribe en problemas de diversa índole que adolecían en el país. Junto a los altos niveles de inseguridad que al inicio de su mandato ya estaban presentes en el país, también se han señalado causas de corte político como origen del anuncio y la estrategia seguida por el Presidente desde 2006. Así, el debate generado en el país sobre la legitimidad de su mandado tras las elecciones ganadas por un estrecho margen al candidato Andrés Manuel López Obrador tan sólo 5 meses antes del anuncio que inició la "guerra", también ha sido visto como un posible causante del anuncio (Campbell y Hansen, 2014).

Independientemente de las causas del anuncio, está claro que desde esa decisión de apertura de la "guerra contra el narcotráfico", los niveles de violencia e inseguridad en México se dispararon drásticamente (Carreón y de la Cruz, 2012; Correa-Cabrera, 2012). Especialmente desde 2008 como resultado del incremento de la guerra entre los propios cárteles por el territorio (Rios, 2014). Y es que el conflicto de narco-violencia rápidamente se extendió a diferentes ámbitos, como la lucha entre diferentes cárteles, dentro de los propios cárteles, entre cárteles y gobierno e incluso entre gobierno y sociedad civil (Campbell y Hansen, 2014). Este clima de inseguridad se manifestó principalmente en un aumento de actos delictivos como secuestros, robos y asesinatos relacionados con las drogas, en muchas ocasiones con una puesta en escena con alta brutalidad y sadismo, hasta tal punto que en gran medida ha sido considerado como un posible ejercicio de terrorismo por las formas de actuar de los grupos delictivos (Campbell y Hansen, 2014).

Quizá el mejor ejemplo de los efectos de esta guerra emprendida contra el narcotráfico sea el número de muertes relacionadas directamente con esta estrategia gubernamental o las actividades de los diferentes cárteles. Se calcula que entre 2006 y 2012, sexenio de Felipe Calderón, se produjeron en México entre 60,000 y 100,000 víctimas de las diferentes acciones violentas sufridas en diferentes zonas de la República (Campbell y Hansen, 2014; Magaloni et al., 2014). En este sentido, no es de extrañar que la inseguridad se convirtiera en el tema de mayor preocupación para la 
ciudadanía mexicana entre 2010 y 2013 (Carreón y de la Cruz, 2012; Rodelo, 2014). Y es que, como recuerdan Magaloni et al. (2014), la actividad del crimen organizado o de las acciones contra los cárteles de la droga, no sólo afecta política a los países y sus gobernantes, sino que sobre todo impacta en la integridad física, el estilo de vida y las propiedades de las personas.

Dentro de las diversas manifestaciones de violencia que la guerra contra el narco presenta en México, la extorsión y el pago del denominado "cobro de piso" ha sido un mal sufrido por buena parte de la sociedad (Carreón y de la Cruz, 2012; Rios, 2014). El hecho de que sea una forma rápida de obtención de dinero para estos grupos delictivos, lo ha convertido en uno de los actos más extendidos (Rios, 2014). Así lo ha puesto de manifiesto la Encuesta Nacional Mexicana sobre Victimización y Percepción de la Seguridad Pública (INEGI, 2013), señalando que este delito fue el segundo en presencia sólo por debajo del robo o asalto en calle o transporte público. Este delito se ceba en especial con aquellos que cuentan con mayor debilidad, como son ciudadanos anónimos y pequeños y medianos empresarios (Carreón y de la Cruz, 2012; Rios, 2014). De hecho, este tipo de acciones delictivas afectó a las dinámicas empresariales hasta el punto de llevar a muchos empresarios a la quiebra, viéndose obligados a cerrar sus negocios y Pymes o incluso a emigrar.

En un contexto de instituciones frágiles como el mexicano (Parker, 2009), los empresarios enfrentan retos como, entre otros, la corrupción (Vassolo et al., 2011) o la acción criminal de las organizaciones criminales (United Nations Office on Drugs and Crime, 2013). En cierta manera, y como señala Correa-Cabrera (2012), el gobierno mexicano ha perdido el monopolio de los impuestos, pues las extorsiones de las organizaciones han generado una especie de doble carga impositiva. El crimen organizado, desafía a los empresarios, quienes no pueden confiar en los derechos de propiedad incluidos en el imperio de la ley, que por lo general carecen de poder de negociación frente a la autoridad de los cárteles del crimen organizado (Parker, 2009) y la burocracia pública (Estrin y Prevezer, 2011). Ante esta situación, los empresarios, consciente o inconscientemente, idean diferentes métodos para responder a las diferentes presiones institucionales, que pueden ser aplicadas tanto ante las presiones de los gobiernos legítimamente establecidos, como ante contextos difíciles por la acción de la delincuencia organizada y la existencia de actos de violencia. Al respecto, Oliver (1991) propone un modelo que abarca varias respuestas estratégicas del empresariado a las presiones institucionales: consentimiento, evasión, desafio y manipulación.

El consentimiento consiste en aceptar las presiones institucionales, a través de estrategias como la adhesión inconsciente a las normas, la imitación voluntaria o evasión consciente y conformidad, como puede ser por ejemplo la aceptación de la extorsión o cobro de piso, bien sea voluntaria o involuntariamente. Por su parte, la evasión implica un intento empresarial para evadir la presión, mediante acciones de inconformismo encubierto (buffering) tras una fachada de aceptación (Pache \& Santos, 2010) o de escape del ámbito de las presiones recibidas (Hirschman, 1970). Esta última táctica es quizá la causante de los cerca de 160 mil desplazados en el interior de la República o hacia al exterior, principalmente EE.UU. (Magaloni et al., 2014), en lo que ha sido denominado como una "nueva" ola de migración mexicana de clase alta 
a los EE.UU. y Canadá (Durin, 2012). En su trabajo, Rios (2014) señala cómo muchos mexicanos se movieron con sus negocios hacia los EE.UU., sobre todo en ciudades fronterizas, donde reabrieron sus negocios para eludir la extorsión.

Por su parte, el desafio es la forma más activa de resistir la presión institucional, que incluye las tácticas como ignorar las reglas y valores institucionales -sobre todo cuando la posibilidad de la aplicación externa de las reglas institucionales es baja-, contradecir las normas impuestas o directamente optar por el ataque como salida agresiva para alejarse de las presiones y expectativas institucionales (Oliver, 1991). Por ejemplo, una respuesta seguida por parte del empresariado en México ante el clima de inseguridad fue la creación organizaciones de autodefensa, paralelos a los servicios públicos como la policía o incluso el ejército (Rios, 2014). Finalmente, la manipulación implica un intento activo para cambiar o ejercer poder sobre las presiones institucionales a través de la cooptación o intento de neutralizar la oposición institucional y aumentar la legitimidad a través del dominio de los componentes que presionan la organización (Henrekson y Sanandaji, 2011; Oliver, 1991). Unas tácticas que buscan un "intento absoluto de cambiar en lugar de escapar" (Hirschman, 1970: 30).

\subsection{Framing de la violencia e inseguridad en México}

Si bien la violencia y actos de inseguridad derivados de la acción de los cárteles y grupos dedicados al narcotráfico en México han estado presentes en las noticias de los medios de comunicación por mucho tiempo, tras el anuncio de Felipe Calderón de inicio de la "guerra contra el narcotráfico" se incrementó la cobertura de estos actos violentos y la presentación de imágenes de esta guerra (Hernández y Rodelo, 2010; Magaloni et al., 2014), en gran medida por el atractivo mediático del tema en cuestión (Rodelo, 2014). Noticias sobre secuestros, asesinatos y otras acciones violentas sufridas por la ciudadanía mexicana y sus diferentes grupos sociales se hicieron habituales en los medios de comunicación internacionales. En gran medida, como recuerda Correa-Cabrera (2012), a través de una cobertura que presentaba la violencia de las drogas en México con una excesiva espectacularización y exageración, como ocurrió en buena parte de los medios de comunicación norteamericanos. Además, en la cobertura periodística internacional de la guerra contra las drogas en México a menudo han destacado los aspectos negativos del contexto institucional (Scott, 2013).

A pesar de esta intensa cobertura del acontecimiento por parte de los medios de comunicación internacionales, son pocos los estudios que hayan abordado esta cobertura informativa, mediante la realización de análisis de contenido que den cuenta de las estrategias seguidas para informar sobre el mismo. Al respecto, recientemente Rodelo (2014) ha sugerido, a través de un análisis de contenido de prensa y televisión mexicanas, que en las en las noticias sobre narcotráfico y delincuencia organizada sobre todo destacó la cobertura de acontecimientos de violencia recibida a nivel individuales (robos, asesinatos, extorsión, etc.) y no tanto un debate en torno al tema del narcotráfico. Por su parte, Portilla (2012) recuerda que la cobertura de los medios de estos asuntos deja que desear, debido en gran medida a la falta de acuerdos claros entre los periodistas sobre qué y cómo informar de estos asuntos (Hernández y Rodelo, 2010). 
El tratamiento informativo particular ofrecido en los medios de comunicación a los acontecimientos ha sido abordado desde la Teoría del Framing o del encuadre, a través del estudio de los encuadres noticiosos utilizados por los periodistas para elaborar sus informaciones (Semetko y Valkenburg, 2000). El framing implica un proceso de selección de "algunos aspectos de la realidad percibida, haciéndolos más sobresalientes en el texto comunicativo, de tal manera que consigan promover una definición del problema particular, una interpretación causal, una evaluación moral y/o una recomendación de tratamiento para el asunto descrito" (Entman, 1993: 52). Dentro del estudio de las rutinas periodísticas, Valkenburg et al. (1999: 550) describen el encuadre mediático como "una forma particular a través de la cual el periodista compone o construye una noticia para optimizar la accesibilidad de la audiencia", que implica una selección de ciertos elementos existentes en el mensaje y una utilización conjunta de los mismos para construir encuadres que dotan de significado al mensaje (de Vreese, 2003).

Un trabajo importante de los investigadores dentro de esta corriente teórica ha sido el de determinar los encuadres existentes en las noticias. Tomando como referencia varios estudios previos, Semetko y Valkenburg (2000) desarrollaron un listado de cinco encuadres que pueden ser utilizados por los periodistas para reportar cualquier temática en sus noticias. Así, existe el encuadre de atribución de responsabilidad que atribuye tanto la causa, como su posible solución, de un asunto o problema a un gobierno, actor o institución; el de interés humano suele plantear la cara humana o un tratamiento emocional a la hora de presentar un asunto o problema; el encuadre de consecuencias económicas que presenta el asunto en términos de las consecuencias económicas a las que tendrá que hacer frente; el de moralidad que enmarca el asunto desde un contexto de principios religiosos o prescripciones morales y, finalmente, el encuadre de conflicto se centra en el conflicto entre individuos, grupos, o instituciones y que a menudo es enfocado en los medios con el objetivo de capturar el interés del público.

Esta propuesta de encuadres ha sido utilizada por diferentes autores en sus investigaciones (de Vreese, 2003), detectándose cómo el uso de los encuadres depende habitualmente del asunto concreto informado y de la idea final que el periodista está interesado en transmitir a la ciudadanía. Al respecto, cabe recordar que las noticias de los medios de comunicación tienen la capacidad de desempeñar un papel importante a la hora de determinar cómo se capta la dinámica entre los grupos sociales que conforman la sociedad, así como el entorno institucional en el que estos se desarrollan (Scott, 2013). Por ello no es raro que regularmente se enfoquen los acontecimientos en las noticias desde el encuadre del conflicto (de Vreese, 2003; Semetko y Valkembug, 2000). Por ejemplo, Eversman (2013) encontró en las noticias de la prensa de EE.UU. dos corrientes incompatibles de discurso entre la reducción de daños y las estrategias de "guerra contra las drogas". El autor detectó una discrepancia respecto a "la forma en que los opositores y partidarios enmarcaron la naturaleza del consumo de drogas, la adicción y la recuperación, afirmaciones que son fundamento de sus argumentos" (Eversman, 2013: 17).

No se han encontrado, sin embargo, estudios sobre la cobertura periodística de la realidad de un grupo social de especial importancia para cualquier México como son los pequeños empresarios y sus empresas (Pymes), y la influencia de la violencia e inse- 
guridad derivada del narcotráfico sobre sus personas y negocios. Por ello, este estudio se plantea las siguientes preguntas de la investigación al respecto de esta cobertura:

PI1: ¿Qué actos violentos contra empresarios de México se reportan en la prensa y cuál es la respuestas de los empresarios ofrecida en las noticias?

PI2: ¿Existen diferencias significativas en la cobertura realizada por la prensa internacional de esta violencia contra empresarios y respuestas de los mismos?

PI3: ¿Qué encuadres noticiosos se utilizan en la prensa internacional para informar sobre actos de violencia en México que afectan a empresarios?

PI4: ¿Existen diferencias significativas en el tratamiento informativo o framing utilizado por la prensa internacional para informar sobre los actos de violencia que afectan a empresarios en México?

PI5:¿Qué relaciones se dan en las noticias entre el tratamiento informativo o framing y los diferentes actos de violencia que afectan a empresarios en México?

\section{Método}

\subsection{Corpus de noticias analizado}

Con el objetivo de responder las preguntas de investigación planteadas, se realizó un Análisis de Contenido de noticias de prensa internacional relativas a la situación de violencia vivida en México. La búsqueda de las unidades de análisis se realizó a través de la base de datos FACTIVA, que permite acceso a noticias numerosos países en todo el mundo (http://www.dowjones.com/factiva/int/espanol.asp). La búsqueda se realizó utilizando como palabras clave "delincuencia", "violencia", "México", "cárteles", "extorsión", "Pymes" y "empresarios". La búsqueda abarcó noticias publicadas entre el 1 de diciembre de 2006 y el 30 de noviembre de 2012, período correspondiente a la presidencia de Felipe Calderón Hinojosa, cuando comenzó la "guerra contra el narcotráfico". Debido a que la base de datos FACTIVA no incluye noticias de la prensa mexicana, para estos periódicos se realizó una búsqueda manual de noticias en los sitios web de algunos de los periódicos mexicanos más influyentes, atendiendo a su número de lectores (García Rubio, 2013): La Jornada, Milenio, El Porvenir, La Prensa y Proceso.

Las búsquedas realizadas en la base de datos y las webs de los periódicos mexicanos arrojaron un total de 4,436 notas. Dentro de este total de noticias, se seleccionaron únicamente las que cubrían hechos concretos relativos al tema principal de investigación: crimen organizado y violencia contra empresarios y sus Pymes. Para ello se realizó un análisis cualitativo consistente en la búsqueda de las palabras clave de forma combinada, con el objetivo de detectar noticias donde se construyera un relato sobre la violencia organizada y la delincuencia, junto a las respuestas de los empresarios. Este procedimiento redujo el número de artículos a 673 que mencionaban al menos un incidente de violencia. De ellos, sólo 200 presentaron al menos una respuesta por parte de un empresario, que fueron las noticias que finalmente se utilizaron como corpus de unidades de análisis. Estas unidades incluyen información sobre violencia real y actos de delincuencia, así como la respuesta de al menos un empresario a la violencia en México y las declaraciones de empresarios, narrando sus respuestas a la violencia, lo que les confiere una alta relevancia para el estudio. 


\subsection{Libro de códigos}

Se creó un libro de códigos para el desarrollo del Análisis de Contenido con los siguientes apartados:

a) País o región de procedencia de la información: Se realizó un listado de todos los países que arrojaron noticias al estudio que fueron agrupados por zonas geográficas, teniendo en cuenta el número de noticias aportadas por cada una de ellas. Del total de noticias seleccionadas para la realización del estudio, $105(52,5 \%)$ procedían de EE.UU., un $26.5 \%$ eran noticias provenientes de periódicos del propio México ( $n$ $=53)$, mientras que otras 42 noticias $(21 \%)$ pertenecían a periódicos de países de la UE. De entre ellos, el grueso venía constituido por noticias de periódicos españoles $(n=29)$, junto a informaciones provenientes del Reino Unido $(n=7)$ y Francia $(n=$ 6). También se encontraron tres noticias de Canadá y una Nueva Zelanda, que no fueron consideradas en la muestra final de 200 noticias dado el pequeño número de artículos periodísticos procedentes de estos países.

b) Tipos de violencia y delincuencia narrada: A partir de los datos sobre violencia y actos de delincuencia registrados en la Encuesta Nacional Mexicana sobre Victimización y Percepción de la Seguridad Pública (INEGI, 2013), se elaboró un listado de 13 posibles tipos de violencia y/o delincuencia y se evaluó su presencia (1) o ausencia (0) en las noticias, y con ellos se realizó un análisis factorial de componentes principales (con rotación ortogonal) a fin de descubrir su estructura latente. La solución factorial $\left(R^{2}=60\right)$, arrojó dos componentes o tipos de delincuencia o violencia: 1) Violencia contra las Pymes: Los actos violentos y criminales que abarcaron este factor fueron: "extorsión directa a Pymes", "robo total o parcial de materiales Pymes", "destrucción de propiedades Pymes" y "robo o asalto a Pymes" $(\alpha=.70)$; 2) Violencia contra los empresarios: Los actos violentos y criminales que compusieron este factor fueron "robo o asalto (en la calle o en transporte público)", "lesiones por agresión física", "secuestro" y "asesinato" $(\alpha=.75)$.

c) Respuestas de los empresarios a la violencia: Partiendo de la propuesta de Oliver (1991) acerca de las posibles respuestas estratégicas subyacentes a las presiones institucionales antes presentada, se elaboró un listado de 12 posibles tácticas de respuesta adoptadas por los empresarios, como por ejemplo "se acepta la extorsión para evitar consecuencias", "se traslada la empresa a otra ciudad del mismo estado" o "se desarrollan acciones violentas de respuesta a los ataques". Con todos los reactivos se realizó un análisis factorial de componentes principales (con rotación ortogonal) que consiguió explicar el 53\% de la varianza total. Sólo se detectó consistencia para tres respuestas de los empresarios: Consentimiento $(\alpha=.82)$, Evasión $(\alpha=.69)$ y Manipulación $(\alpha=.83)$.

d) Encuadres noticiosos: Se utilizó la escala desarrollada por Semetko y Valkenburg (2000), si bien sólo los encuadres relativos a moralidad, conflicto y consecuencias económicas. En total, se trabajaron con 10 de los 20 ítems con que originalmente cuenta la escala de encuadres noticiosos, una reducción debida a que sólo se utilizaron 3 de los 5 encuadres posibles. Un análisis factorial de componentes principales (con rotación ortogonal) extrajo tres factores reconocibles como los encuadres noticiosos previstos $\left(R^{2}\right.$ $=70$ ). Se calculó la consistencia interna de cada uno de estos indicadores, arrojando 
para todos los encuadres una fiabilidad aceptable: para el de conflicto $(\alpha=.81)$, para el de consecuencias económicas $(\alpha=.85)$ y para el de moralidad $(\alpha=.68)$.

\subsection{Procedimiento y fiabilidad}

Para la realización del estudio se contrató a dos estudiantes de doctorado bilingües (español-inglés), quienes realizaron la codificación de forma separa de los 204 artículos usando un libro de códigos. Ambos estudiantes fueron entrenados para utilizar el libro de códigos, pero no se les reveló ni las preguntas ni el propósito de la investigación. Posteriormente se realizó el chequeo de la fiabilidad del Análisis de Contenido o prueba de acuerdo interjueces (intercoder reliability). Para ello se utilizó de forma aleatoria una submuestra de 50 noticias, que fueron nuevamente codificadas. La Kappa de Cohen arrojó un valor promedio de .90, por lo que se considera el estudio fiable (Krippendorff, 1990).

\section{Análisis de los resultados}

Para responder la primera de las preguntas de investigación planteadas, acerca de ¿Qué actos violentos contra empresarios de México se reportan en la prensa y cuál es la respuestas de los empresarios ofrecida en las noticias?, se realizó un análisis descriptivo de los tipos de violencia y respuestas empresariales detectadas. Así, se puede observar cómo la acción violenta más reportada en general por los periódicos estudiados es la violencia individual o contra el propio empresario $(\mathrm{M}=.58 ; \mathrm{DT}=.34)$, frente a los actos de violencia contra las Pymes o empresas que arrojó una menor presencia $(\mathrm{M}=.21 ; \mathrm{DT}=.28)($ Ver datos en Tabla 1$)$. Además, aunque la presencia de ambos tipos de violencia en las noticias correlacionaba, $\mathrm{r}(198)=.53, \mathrm{p}=.000$, la prevalencia de la violencia contra los empresarios frente a la sufrida en sus negocios es estadísticamente significativa, $\mathrm{t}(199)=17.458, \mathrm{p}=.000, \mathrm{~d}=1.19$. Ello permite concluir cómo la prensa internacional tiende a presentar más actos violentos contra empresarios cometidos en México que contra sus propias empresas. En cuanto a las respuestas de los empresarios a estas presiones, en general se reportaron más acciones de consentimiento o aceptación $(\mathrm{M}=.22 ; \mathrm{DT}=.37)$ que de manipulación $(\mathrm{M}=$ .18 ; $\mathrm{DT}=.33)$ o evasión $(\mathrm{M}=.13$; $\mathrm{DT}=.26)$. Sin embargo, estas diferencias de presencia en las noticias fueron únicamente tendenciales a nivel estadístico, $\mathrm{F}(2,378)=$ $2.982, \mathrm{p}=.055, \eta^{2}$ parcial $=.015$.

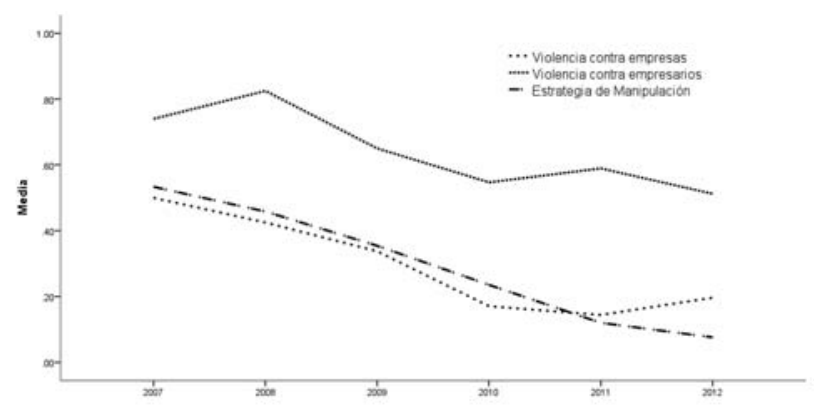

Figura 1. Presencia en las noticias de los tipos de violencia y la estrategia de manipulación a lo largo de los años del Sexenio 2006-2012 
$\mathrm{Al}$ analizar la presencia de los diferentes tipos de violencia y respuestas de los empresarios a las mismas a lo largo de los años estudiados, correspondientes al sexenio presidencial de Felipe Calderón, sólo se detectaron diferencias estadísticamente significativas para la presencia de actos de violencia contra empresas, $F(5,194)=5.288$, $p=.000, \eta^{2}=.120$, y de respuestas de manipulación por parte de los empresarios, $F(5$, $194)=6.885, p=.000, \eta^{2}=.151$. También se detectaron diferencias, aunque tendenciales, en la presencia en las noticias de la violencia contra los propios empresarios, $F(5,194)=2.000, p=.080, \eta^{2}=.049$. Como se puede observar en la Figura 1, tanto para la violencia contra las empresas como las repuestas de manipulación se detectó una mayor presencia en las noticias en el primer año estudiado, que fue decayendo conforme avanzó el sexenio. Solo se detecta un repuntó de la cobertura de violencia contra empresas en 2011 y 2012. Por su parte, la mayor cobertura de la violencia contra empresarios se oreció en 2008, decayendo posteriormente su presencia salvo por el leve repunte detectado en 2011.

Asimismo, el estudio planteó la pregunta de si ¿Existen diferencias significativas en la cobertura realizada por la prensa internacional de esta violencia contra empresarios y respuestas de los mismos? Para dar respuesta a esta pregunta, se realizó un análisis multivariado de varianza (MANOVA) con los actos violentos y criminales (violencia contra las Pymes y violencia contra los empresarios) y las respuestas estratégicas de los empresarios como variables dependientes, mientras que los países y/o regiones constituyeron la variable independiente. Los resultados muestran diferencias entre los países y/o regiones a nivel multivariado, $\lambda$ Wilks $=.757, F(10,386)$ $=5.768, p=.000, \eta^{2}=.130$, lo que indica que los EE.UU., México y la UE no presentaban el mismo efecto combinatorio sobre violencia reportada y las respuestas de los empresarios a la misma.

Tabla 1. Cobertura de los tipos de violencia y estrategias seguidas por los empresarios en las noticias de EE.UU., México y UE

\begin{tabular}{|l|c|c|c|c|}
\hline \multirow{2}{*}{\multicolumn{1}{c}{$\begin{array}{c}\text { Tipos de violencia vivida y } \\
\text { estrategias seguidas }\end{array}$}} & \multicolumn{3}{|c|}{ País o región } & \multirow{2}{*}{ Total } \\
\cline { 2 - 5 } & EE.UU. & México & UE & \\
\hline Violencia contra las Pymes & $.23(.29)^{*}$ & $.12(.25)^{*}$ & $.25(.26)^{*}$ & $.21(.28)$ \\
\hline Violencia contra los empresarios & $.65(.32)^{* * *}$ & $.43(.37)^{* * *}$ & $.61(.28)^{* * *}$ & $.58(.34)$ \\
\hline Evasión & $.13(.22)^{* * *}$ & $.23(.36)^{* * *}$ & $.02(.07)^{* * *}$ & $.13(.26)$ \\
\hline Consentimiento & $.15(.33)^{*}$ & $.23(.41)^{*}$ & $.32(.41)^{*}$ & $.21(.37)$ \\
\hline Manipulación & $.20(.33)$ & $.16(.32)$ & $.16(.35)$ & $.18(.33)$ \\
\hline
\end{tabular}

Nota: $N=200$. Los valores entre paréntesis representan la Desviación Estándar. Las diferencias son estadísticamente significativas a nivel $* * * p<.001 ; * p<.5$

A nivel univariado, también se detectaron diferencias estadísticamente significativas en los actos violentos y dos de las estrategias empresariales informadas (Ver Tabla 1). En cuanto a la cobertura de actores de violencia contra las propias Pymes, se detectaron diferencias significativas, $F(2,197)=3.681, p=.027, \eta^{2}=.036$. La prueba post-hoc de Scheffé realizada mostró que las noticias de la UE $(M=.25 ; D T=.26) \mathrm{y}$ 
de EE.UU. $(M=.23 ; D T=.29)$ reportaban más este tipo de violencia en conjunto que las mexicanas $(M=.12 ; D T=.25)$. En cuanto a la violencia contra los propios empresarios, también se detectaron diferencias estadísticamente significativas, $F(2$, $197)=8.865, p=.000, \eta^{2}=.083$, entre los diferentes países y/o regiones. La prueba post-hoc de Scheffé realizada indicó que los periódicos de la UE $(M=.61 ; D T=.28)$ y de EE.UU. $(M=.65 ; D T=.28)$ presentaron una intensidad similar de cobertura de estos hechos violentos, más fuerte en ambos casos que la cobertura ofrecida por los periódicos de México $(M=.43 ; D T=.37)$.

En cuanto a las reacciones de los empresarios ante estos actos violentos reportadas por los medios, se detectaron diferencias estadísticamente significativas en el caso de la estrategia de evasión, $F(2,197)=8,744, p=.000, \eta^{2}=.082$. La prueba post-hoc de Scheffé realizada mostró que los periódicos de México $(M=.23 ; D T=.36)$ presentaron más esta estrategia que los periódicos de los EE.UU. $(M=.13 ; D T=.22)$ y los de la UE $(M=.02 ; D T=.07)$ informaron en menor medida de esta estrategia empresarial. También se detectaron diferencias en la cobertura de la estrategia del consentimiento o aceptación, $F(2,197)=3.251, p=.041, \eta^{2}=.032$, que atendiendo a la prueba post-hoc de Scheffé, se presentaron entre los periódicos de la región UE ( $M=$ $.32 ; D T=.41)$, los que adoptaron esta estrategia más frecuentemente que los de México $(M=.23 ; D T=.41)$ y los de EE.UU. $(M=.15 ; D T=.33)$. Por último, los resultados no mostraron una diferencia estadísticamente significativa para la cobertura de la estrategia empresarial de manipulación, $F(2,197)=0.394, p=.675, \eta^{2}=.004$.

Por otra parte, la pregunta de investigación tercera cuestionaba ¿Qué encuadres noticiosos se utilizan en la prensa internacional para informar sobre actos de violencia en México que afectan a empresarios? Para responderla, se analizó la presencia de los encuadres de conflicto, moralidad y consecuencias económicas (Semetko y Valkenburg, 2000). El estudio descriptivo de estos tres encuadres muestra cómo el de consecuencias económicas fue el más utilizado $(M=.38 ; D T=.43)$ para elaborar la información sobre la violencia sufrida por los empresarios y sus respuestas ante la misma. En segundo lugar se situó la presencia en las noticias del encuadre de moralidad $(M=.20 ; D T=.30)$, mientras que el encuadre de conflicto fue el menos utilizado $(M=.18 ; D T=.31)$ por los periodistas a nivel internacional para presentar este tipo de informaciones en los periódicos (Ver datos en Tabla 2). La prevalencia del encuadre de consecuencias económicas sobre los demás fue clara en la muestra analizada, $F(2,339)=21.701, p=.000, \eta^{2}$ parcial $=.098$.

En el caso de la pregunta de investigación cuarta, en el estudio se cuestionó si ¿Existen diferencias significativas en el tratamiento informativo o framing utilizado por la prensa internacional para informar sobre los actos de violencia que afectan a empresarios en México? Para dar respuesta a esta duda se realizó un análisis multivariado de varianza (MANOVA), utilizando como variables dependientes los tres encuadres noticiosos estudiados y el país y/o región de la noticia como variable independiente. La prueba arrojó la existencia de un efecto principal significativo entre las variables independientes, $\lambda$ Wilks $=.789, F(6,390)=8.158, p=.000, \eta_{\text {parcial }}^{2}=$ .112. Esta interacción indica que el efecto conjunto de los tres encuadres de noticias no es el mismo en los EE.UU., México y la UE. Con el objetivo de determinar las di- 
ferencias a nivel univariado, se realizaron análisis de varianza (ANOVA) para cada uno de los encuadres noticiosos.

Tabla 2. Utilización de los encuadres noticiosos en las noticias de EE.UU., México y UE.

\begin{tabular}{|l|c|c|c|c|}
\hline \multirow{2}{*}{ Encuadres Noticiosos } & \multicolumn{3}{|c|}{ País o región } & \multirow{2}{*}{ Total } \\
\cline { 2 - 5 } & \multirow{2}{*}{ EE.UU. } & México & UE & \\
\hline Conflicto & $.23(.33)^{*}$ & $.08(.24)^{*}$ & $.18(.30)^{*}$ & $.18(.31)$ \\
\hline Consecuencias Económicas & $.48(.43)^{* * *}$ & $.38(.43)^{* * *}$ & $.16(.32)^{* * *}$ & $.38(.43)$ \\
\hline Moralidad & $.15(.24)^{* *}$ & $.32(.36)^{* *}$ & $.17(.31)^{* *}$ & $.20(.30)$ \\
\hline
\end{tabular}

Nota: $N=200$. Los valores entre paréntesis representan la Desviación Estándar. Las diferencias son estadísticamente significativas a nivel $* * * p<.001 ; * * p<.01 ; * p<.5$

Como se observa en la Tabla 2, los resultados del uso del encuadre de conflicto son estadísticamente significativos, $F(2,197)=4,298, p=.015, \eta^{2}=.042$. En este sentido, la prueba post-hoc de Scheffé permitió observar que las diferencias se presentaban entre todos los países y/o regiones. Así, la prensa de EE.UU. fue la que en mayor medida utilizó en sus noticias el encuadre de conflicto $(M=.23 ; D T=.33)$, seguido por los periódicos de la UE $(M=.18 ; D T=.30)$. Finalmente, las noticias de México fueron las que en menor medida presentaron este $(M=.08 ; D T=.24)$. También se detectó una diferencia significativa en el uso del encuadre de consecuencias económicas, $F(6,390)=8.994, p=.000, \eta^{2}=.084$. La prueba post-hoc de Scheffé reveló que las noticias de EE.UU. $(M=.48 ; D T=.43)$ eran las que más utilizaban este encuadre, seguidas de las noticias de medios mexicanos $(M=.38 ; D T=.43)$ y de la UE $(M=.16$; $D T=.32)$. Por último, se encontró una diferencia estadísticamente significativa para el uso del encuadre de moralidad, $F(6,390)=6.538, p=.002, \eta 2=.062)$. La prueba post-hoc de Scheffé permitió observar que las noticias de periódicos mexicanos $(M=$ $.32 ; D T=.36)$ utilizan este encuadre con mayor frecuencia que las de la prensa de EE.UU. $(M=.15 ; D T=.24)$ o de la UE $(M=.17 ; D T=.31)$.

Tabla 3. Correlaciones en el uso de encuadres noticiosos y estrategias de los empresarios Nota:

\begin{tabular}{|l|c|c|c|}
\hline \multirow{2}{*}{\begin{tabular}{c}
\multirow{2}{*}{$\begin{array}{c}\text { Tipos de violencia vivida y } \\
\text { estrategias seguidas }\end{array}$} \\
\cline { 2 - 4 }
\end{tabular}} & \multicolumn{3}{c|}{ Encuadres Noticiosos } \\
\cline { 2 - 4 } & Conflicto & $\begin{array}{c}\text { Consecuencias } \\
\text { Económicas }\end{array}$ & Moralidad \\
\hline Violencia contra las Pymes & $.44^{* * *}$ & -.03 & .14 \\
\hline Violencia contra los empresarios & $.32^{* * *}$ & .12 & .10 \\
\hline Evasión & .06 & $.43^{* * *}$ & $.20^{* *}$ \\
\hline Consentimiento & $.24^{* * *}$ & .13 & $.44^{* * *}$ \\
\hline Manipulación & $.35^{* * *}$ & -.12 & $.001 ; * * p<.01$ \\
\hline
\end{tabular}

Finalmente, la última pregunta de investigación planteaba la duda respecto a ¿Qué relaciones se dan en las noticias entre el tratamiento informativo o framing y los diferentes actos de violencia que afectan a empresarios en México? Para responderla se 
calcularon las correlaciones entre las diferentes variables del estudio (Ver Tabla 3). Los resultados permiten observar una estrategia de vincular las noticias enfocadas desde el conflicto con actos de violencia contra empresas, $r(198)=.44, p=.000$, violencia contra los empresarios, $r(198)=.32, p=.000$, y estrategias empresariales como el consentimiento o aceptación, $r(198)=.24, p=.001$, y la manipulación, $r(198)=.35, p$ $=.000$. En cuanto al encuadre de consecuencias económicas, éste solía aparecer en noticias donde se informaba de la estrategia de evasión, $r(198)=.43, p=.000$. Por su parte, el encuadre de moralidad tendía a aparecer en informaciones sobre evasión de empresarios, $r(198)=.20, p=.004$, o bien de manipulación sobre los gobiernos, $r(198)=.44, p=.000$.

\section{Discusión y conclusiones}

El objetivo de este trabajo fue el de investigar las diferencias en la cobertura presentada en los periódicos internacionales de las acciones violentas contra los empresarios en México y las estrategias de respuesta de éstos ante la situación de inseguridad derivada de la guerra contra el narcotráfico. En general, se advierte que los periódicos de EE.UU. y la UE informan más que los de México sobre actos de violencia contra infraestructuras Pymes y los propios empresarios. Parece haber, por tanto, una estrategia de los propios medios mexicanos para reducir la cobertura de este tipo de actos violentos, que además se hizo más fuerte conforme fue avanzando el sexenio estudiado. Por el contrario, los periódicos mexicanos dominaron en la presentación de la evasión o escape como respuesta de los empresarios ante estos actos de violencia e inseguridad sufrida, muchas veces trasladándose a otras zonas del país, otras huyendo al extranjero. Sin embargo, el consentimiento que en muchas ocasiones se tuvo que hacer ante presiones o extorsiones sufridas fue claramente dominante en la cobertura realizada por la UE.

Parece advertirse que los periódicos de EE.UU. y la UE, junto con la cobertura habitual que se puede hacer de acontecimientos conflictivos a nivel internacional como es la guerra contra el narcotráfico en México, también se preocuparon por dar una imagen sobre una zona donde las inversiones empresariales de ambos países son muy fuertes. Esta acción informada (violencia y crimen) parece ser relevante para los negocios internacionales, ya que los EE.UU. y la UE son las dos regiones del mundo con relaciones comerciales importantes en México, principalmente en inversión extranjera directa y comercio (Comisión Europea, 2013). La cobertura de las noticias en los medios de comunicación también podría dar a entender que la violencia contra infraestructura y sobre todo contra los propios empresarios, aspecto destacado en los periódicos de EE.UU. y la UE, es la forma en la cual los negocios operan en México, debiendo aceptar en gran medida las presiones a través de una estrategia de consentimiento.

De manera contraria, la prensa mexicana, quizá por la falta de acuerdos claros entre los periodistas sobre qué y cómo informar de estos asuntos señalada por Hernández y Rodelo (2010), tendieron a "enfriar" la realidad, ofreciendo una visión más leve de la violencia contra empresarios y sus negocios en las noticias. En cualquier caso, su mayor cobertura fue de los actos violentos sufridos directamente por los empresarios 
de forma personal, que ya no solo ponían en peligro sus negocios e inversiones, sino su propia integridad física o la de las personas de su entorno. En este contexto, es interesante observar cómo en México la estrategia de evasión seguida por parte de los empresarios fue la más utilizada entre los periodistas para reflejar los efectos de los actos de violencia e inseguridad. Una representación de derrota ante el narcotráfico, que se une al discurso de buena parte de la sociedad acerca de que la intervención gubernamental de lucha contra los cárteles había fracasado (Magaloni et al., 2014) o incluso con la percepción de México como un "estado fallido" que se extendió a nivel internacional (Correa-Cabrera, 2012; Campbell y Hansen, 2014).

El análisis de la presencia de los diferentes encuadres noticiosos en las noticias deja entrever cómo, en términos generales, la prensa internacional destacó las consecuencias económicas ocasionadas por la violencia e inseguridad contra los empresarios en México, fue el que mayor riqueza argumentativa ofreció en las noticias analizadas. Frente a informaciones centradas en la evasión o escape de los empresarios como principal consecuencia económica de este clima de inseguridad, las informaciones que presentaban conflicto o enfrentamiento entre actores se enfocaban en la violencia recibida por los empresarios o directamente sus negocios. Y, como era de esperar, este conflicto implicaba o bien una estrategia de consentimiento donde el empresario aparece como el vencido o derrotado en el conflicto, o bien una manipulación donde el empresario toma el papel de actor atacante que trata de rechazar las presiones recibidas.

Por otra parte, los resultados reflejan en gran medida diferentes estrategias por parte de los distintos países y/o regiones. El predominio del encuadre de conflicto en los medios de comunicación de EE.UU. y la UE sugiere que los periodistas en estas regiones parecen adoptar diferentes perspectivas en la interpretación de la violencia organizada y la delincuencia en México. Por ejemplo, los artículos estadounidenses tendían en presentar temas relacionados con la corrupción de funcionarios mexicanos y el aumento de la violencia en los estados fronterizos entre EE.UU. y México, como resultado de las acciones de los carteles de la droga en México. Un perfecto enfoque para presentar a México como ese "estado fallido" antes mencionado (Campbell y Hansen, 2014).

Por su parte, las noticias procedentes de EE.UU. y México mostraban más el encuadre económico que las de la UE. Se puede deducir que los costos de la "guerra contra los cárteles de la droga" y sus consecuencias económicas, como la pérdida de empresarios en México, parecen ser un tema central común para los periodistas en México y EE.UU.. Las noticias de la muestra tienden a discutir los aspectos económicos de la delincuencia organizada y la violencia en México. Otras agencias de noticias enfatizan los costos de la "guerra contra los cárteles de la droga" para los gobiernos de EE.UU. y México, como los mil seiscientos millones de dólares destinados por EE.UU a la Iniciativa Mérida de lucha contra el narco en México (Departamento de Estado de EE.UU., 2013). Por su parte, periódicos mexicanos como La Jornada y El Norte refieren en sus noticias los costos que representa la delincuencia organizada para la sociedad civil, como los pagos de extorsión (cobros de piso) o el cierre de Pymes en México. 
Finalmente, el encuadre de moralidad fue particularmente evidente en las noticias de México, que sugerían una prescripción social para la forma de enfrentar el conflicto entre las disposiciones institucionales legítimas e ilegítimas en el país. Los hallazgos sugieren que los miembros de la sociedad civil en instituciones frágiles siguen siendo vulnerables a la violencia y el crimen (Parker, 2009). Esta tendencia de la prensa mexicana a enfocar en términos de moralidad en el cambio que el aumento de la delincuencia organizada y la violencia parecen haber tenido en el modus operandi de la sociedad civil. Este clima favoreció la presentación de alternativas para enfrentar -o evitar- la violencia y la delincuencia creándose normas sociales que en gran medida pueden estar presentes en este tipo de informaciones con encuadre de moralidad.

\section{Referencias bibliográficas}

AMERICAN CHAMBER OF COMMERCE OF MEXICO (2010): Foreign Direct Investment in Mexico: Is Your Investment Safe? http://www.amcham.org.mx /wcwebcontent/webcontentpage.aspx?contentid=11171 [Consulta: 4 de mayo de 2014].

CAMPBELL, Howard \& HANSEN, Tobin (2014): "Is Narco-Violence in Mexico Terrorism?" Bulletin of Latin American Research, vol. 33, $\mathrm{n}^{\circ} 2$. Oxford, John Wiley \& Sons Ltd, pp. 158-173. Doi: 10.1111/blar.12145

CARREÓN GUILLÉN, Javier y DE LA CRUZ LUGARDO, Pedro Isnardo (2012): "La lucha actual contra la delincuencia organizada en México". Barataria: revista castellano-manchega de ciencias sociales, $\mathrm{n}^{\mathrm{o}}$ 14. Toledo, Asociación castellanomanchega de Sociología, pp. 59-74.

COMISIÓN EUROPEA (2013): Trade Countries and Region -Mexico: http://ec.europa.eu/trade/policy/countries-and-regions/countries/mexico/ [Consulta: 2 de mayo de 2014].

CORREA-CABRERA, Guadalupe (2006): “The Spectacle of Drug Violence: American Public Discourse, Media, and Border Enforcement in the Texas-Tamaulipas Border Region during Drug-War Times". Norteamérica, vol. 7, $\mathrm{n}^{\circ} 2$. Ciudad de México, Centro de Investigaciones sobre América del Norte, UNAN, pp .199-220.

DE VREESE, Claes (2003): Framing Europe: Television news and European integration. Amsterdam, Aksant Academic Publishers.

DEPARTAMENTO DE ESTADO DE EE.UU. (2013): Merida Initiative. http://www.state.gov/j/inl/merida/ [Consulta: 2 de mayo de 2014].

DURIN, Séverine (2012): "Los que la guerra desplazó: familias del noreste de México en el exilio". Desacatos, n ${ }^{\circ}$ 38. México, CIESAS, pp. 29-42.

ENTMAN, Robert (1993): "Framing: Toward clarification of a fractured paradigm". Journal of communication, vol. $43, \mathrm{n}^{\circ}$ 4. Hoboken, EE.UU., Wiley-Blackwell, pp. 51-58. Doi: 10.1111/j.1460-2466.1993.tb01304.x

ESTRIN, Saul \& PREVEZER, Martha (2011): "The role of informal institutions in corporate governance: Brazil, Russia, India, and China compared". Asia Pacific 
journal of management, vol. 28, $\mathrm{n}^{\circ}$ 1. Nueva York, Springer, pp. 41-67. Doi: 10.1007/s10490-010-9229-1

EVERSMAN, Michael (2013): "Harm Reduction and Illicit Drugs in US Newspapers". Journal of Drug Policy Analysis, vol. 6, n 1 . Walter de Gruyter, pp. 1-21. doi: $10.1515 /$ jdpa-2012-0005

GARCÍA RUBIO, Claudia (2013): "Radiografía de la prensa diaria en México en 2010". Comunicación y Sociedad, n 20. Guadalajara, Universidad de Guadalajara, pp. 65-93.

HENREKSON, Magnus y SANANDAJI, Tino (2011): “The interaction of entrepreneurship and institutions". Journal of Institutional Economics, vol. 7, $\mathrm{n}^{\circ} 1$. Cambridge, Cambridge University Press, pp. 47-75. Doi: 10.1017/S1744137410000342

HERNÁNDEZ, María Elena y RODELO, Frida (2010): "Dilemas del periodismo mexicano en la cobertura de 'la guerra contra el narcotráfico': ¿periodismo de guerra o de nota roja?", en RODRÍGUEZ, Zeyda: Entretejidos comunicacionales. Aproximaciones a objetos y campos de la comunicación. Guadalajara, Universidad de Guadalajara, pp. 193-228.

HIRSCHMAN, Albert (1970): Exit, voice, and loyalty: Responses to decline in firms, organizations, and states. Cambridge, Harvard University Press.

INEGI (2013): Encuesta nacional de victimización y percepción sobre seguridad pública (ENVIPE) (No. 364.101). México, Instituto Nacional de Estadística y Geografía.

KRIPPENDORFF, Klaus (1990): Metodología del análisis de contenido. Teoría y práctica. Barcelona, Paidós.

MAGALONI, Beatriz; DÍAZ-CAYEROS, Alberto; \& ROMERO, Vidal (2014): "The Mexican War on Drugs: Crime and the Limits of Government Persuasion". International Journal of Public Opinion Research (en prensa). Oxford, Oxford Univ Press. doi: 10.1093/ijpor/edu009

MUÑIZ, Carlos; RAMÍREZ, Jacobo; MURGÁN, Andrea; \& CASTILLO, José Manuel (2009): "Framing investment in Mexico: An exploratory content analysis of the news frames of the main Spanish companies in the Mexican press". European Journal of International Management, vol. 3, $\mathrm{n}^{\circ} 1$. Ginebra, Inderscience Enterprises Ltd, pp. 216-231. Doi: 10.1504/EJIM.2009.024323

OLIVER, Christine (1991): "Strategic responses to institutional processes". Academy of Management Review, vol. 16, $\mathrm{n}^{\circ} 1$. Nueva York, Academy of Management, pp. 145-179. Doi: 10.5465/AMR.1991.4279002

PACHE, Anne Claire \& SANTOS, Filipe (2010): "When worlds collide: The internal dynamics of organizational responses to conflicting institutional demands". Academy of Management Review, vol. 35, $\mathrm{n}^{\circ}$ 3. Nueva York, Academy of Management, pp. 455-476.

PARKER, Simon (2009): The economics of entrepreneurship. Cambridge, Cambridge University Press. 
PORTILLA, Radamanto (2012): "Propuesta metodológica para el análisis de los encuadres periodísticos en la cobertura del narcotráfico en México". Revista Iberoamericana de Comunicación, $\mathrm{n}^{\circ}$ 22. Ciudad de México, Universidad Iberoamericana, pp. 43-72.

RIOS, Viridiana (2014): "Security Issues and Immigration Flows: Drug-Violence Refugees, the New Mexican Immigrants". Latin American Research Review, vol. 49, $n^{\circ}$ 3. Montreal, Latin American Studies Association, (en prensa).

RODELO, Frida (2014): "El tráfico de drogas y la delincuencia organizada en medios mexicanos de distinto alcance y sector". Global Media Journal México, vol. 11, n 21. Monterrey, Tecnológico de Monterrey, pp. 50-66.

SCOTT, Richard (2013): Institutions and Organizations. Ideas, Interests, and Identities. Londres, Sage.

SEMETKO, Holli \& VALKENBURG, Patti (2000): "Framing European politics: A content analysis of press and television news". Journal of communication, vol. 50, $\mathrm{n}^{\circ}$ 2. Hoboken, EE.UU., Wiley-Blackwell, pp. 93-109. Doi: 10.1111/j.14602466.2000.tb02843.x

UNITED NATIONS OFFICE ON DRUGS AND CRIME (2013): Transnational organized crime: the globalized illegal economy. http://www.unodc.org/toc/en /crimes/organized-crime.html [fecha de consulta: 4 de mayo de 2014].

VALKENBURG, Patti; SEMETKO, Holli; \& DE VREESE, Claes (1999): “The effects of news frames on reader's thoughts and recall". Communication Research, vol. 26, $\mathrm{n}^{\circ}$ 5. Thousand Oaks, USA, SAGE, pp. 550-569.

VASSOLO, Roberto; DE CASTRO, Julio; \& GOMEZ-MEJIA, Luis (2011): "Managing in Latin America: Common issues and a research agenda". The Academy of Management Perspectives, vol. 25, $\mathrm{n}^{\circ}$ 4. Nueva York, Academy of Management, pp. 22-36. doi: 10.5465/amp.2011.0129 